\title{
Surgical Approaches for Vestibular Schwannoma
}

\author{
Alex D. Sweeney • Matthew L. Carlson • \\ Moneeb Ehtesham • Reid C. Thompson • \\ David S. Haynes
}

Published online: 10 September 2014

(C) Springer Science+Business Media New York 2014

\begin{abstract}
Despite the emergence of multiple management options for patients with vestibular schwannomas, surgery remains an important part of the treatment algorithm. The retrosigmoid, translabyrinthine, and middle fossa craniotomies are the three most commonly employed approaches for microsurgical resection. Each has inherent advantages and limitations, which are described in this article. Additionally, we present indications and procedural highlights for each.
\end{abstract}

Keywords Vestibular schwannoma - Acoustic neuroma . Skull base tumor microsurgery $\cdot$ Surgical approaches

\section{Introduction}

Vestibular schwannomas (VS) are benign, generally slowgrowing tumors that arise from the vestibular portion of the

A. D. Sweeney · M. L. Carlson · R. C. Thompson ·

D. S. Haynes $(\square)$

Department of Otolaryngology-Head and Neck Surgery, The Bill

Wilkerson Center for Otolaryngology \& Communication

Sciences, Vanderbilt University Medical Center, 7209 Medical

Center East, South Tower 1215 21st Avenue South, Nashville,

TN 37232-8605, USA

e-mail: david.haynes@vanderbilt.edu

A. D. Sweeney

e-mail: alex.d.sweeney@vanderbilt.edu

M. L. Carlson

e-mail: matthew.1.carlson@vanderbilt.edu

R. C. Thompson

e-mail: reid.thompson@vanderbilt.edu

M. Ehtesham - R. C. Thompson - D. S. Haynes

Department of Neurological Surgery, Vanderbilt University

Medical Center, Nashville, TN 37232, USA

e-mail: moneeb.ehtesham@vanderbilt.edu eighth cranial nerve. The majority of patients present with sporadic unilateral lesions during the fourth to sixth decade of life after experiencing asymmetrical hearing loss and tinnitus, while approximately $5 \%$ develop bilateral VS, the hallmark of Neurofibromatosis type 2.

Current treatment options for small to medium-sized tumors include microsurgical resection and stereotactic radiosurgery. In most centers, surgery remains the preferred treatment modality for patients with large tumors $(>3 \mathrm{~cm})$ or those with symptoms attributable to mass effect. Within the last two decades, evidence from natural history studies has demonstrated that a significant number of small tumors remain quiescent for extended periods of time, leading to the consideration of an initial "wait-andscan" management option. With increasing use of less invasive management strategies, outcomes following microsurgical resection have become more heavily scrutinized. It is necessary that the surgical team understand the advantages and limitations of each surgical approach in order to optimize surgical exposure and limit morbidity.

While many approaches to the internal auditory canal and cerebellopontine angle have been described, the translabyrinthine, middle fossa, and retrosigmoid craniotomies remain the most commonly employed. The choice between these options is largely dictated by tumor size and location, level of preoperative hearing, patient functional status, and surgeon preference. The retrosigmoid approach provides the most versatile corridor, offering a panoramic view of the posterior fossa for large tumors and the possibility of hearing preservation for small, medially based tumors. The translabyrinthine approach is generally used for patients with non-serviceable hearing or in cases when hearing preservation is not realistically feasible. Finally, the middle fossa craniotomy is reserved for small tumors confined to the internal auditory canal in patients with 
functional preoperative hearing. We provide an in-depth review of these three approaches, highlighting indications, controversies, surgical nuances, and outcomes.

\section{Vestibular Schwannoma Surgery: A Historical Perspective}

The management of VS has evolved considerably over the past century. Initially, the diagnosis of a tumor was suspected based on clinical symptoms and confirmed on autopsy. Early attempts at surgical extirpation suffered from limitations in exposure, hemostasis, and anesthetic delivery. By some accounts, perioperative mortality was as high as $80 \%$ at the turn of the twentieth century [1]. The subsequent efforts of Harvey Cushing and Walter Dandy toward refining the suboccipital craniotomy and tumor dissection techniques led to dramatic reductions in this percentage. In the 1960s, William House, widely considered to be the father of neurotologic surgery, pioneered the operative microscope for tumor dissection resulting in a reduction in associated cranial nerve injury. Jointly with his neurosurgical colleagues, the translabyrinthine and middle cranial fossa approaches to the internal auditory canal and cerebellopontine angle were also popularized [2].

As the risk of perioperative mortality fell, microsurgery began to focus increasingly on minimizing morbidity. In particular, operative efforts sought not only to create the exposure necessary for a safe, complete resection, but also to reliably preserve facial nerve function and, potentially, hearing. Over time, this shift has led to the incorporation of multiple disciplines into the contemporary care of patients with VS. Skull base centers have emerged, which offer the combined expertise of neurotologists, head and neck surgeons, neurosurgeons, neurologists, radiation oncologists, facial plastic surgeons, audiologists, neurophysiologists, anesthesiologists, and physical therapists.

\section{General Indications, Limitations and Risks}

The optimal management strategy for a newly diagnosed VS remains controversial. In general, options include microsurgical resection, stereotactic radiation, and observation with a "wait-and-scan" approach. Historically, surgical resection has been the treatment modality of choice. However, the advent and wide usage of contrast enhanced magnetic resonance imaging (MRI) has led to relatively earlier tumor diagnosis than was previously possible [3]. Compounded with the finding that tumor growth is not always guaranteed or predictable, non-surgical management strategies have materialized [4, 5•]. Radiation has proven to be effective in controlling tumor growth in many cases and has been used with increasing frequency over the past few decades. While somewhat controversial, most clinicians reserve single fraction radiation for non-cystic, growing tumors less than $3 \mathrm{~cm}$ in the greatest posterior fossa dimension $[6 \bullet \bullet, 7 \bullet \bullet]$. Additionally, a "wait-and-scan" strategy has been born from natural history studies showing that many small tumors remain quiescent for extended periods of observation [4], and that growth is generally slow when it occurs. Adding to this management dilemma, recent reports suggest that long-term quality of life for VS patients

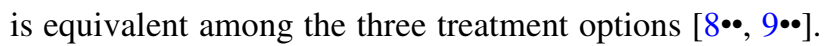

Despite the respective value of "wait-and-scan" management and stereotactic radiation for VS, microsurgical resection remains an important part of the treatment algorithm. When tumor mass results in moderate brainstem or cerebellum compression, intervention is generally warranted. The consequences of persistent tumor growth beyond this point can include progressive symptoms of mass effect including trigeminal neuropathy, cerebellar ataxia, and hydrocephalus. Given the limitations and risks of radiation therapy in this setting, post-radiation tumor swelling in particular, surgical management becomes a necessary consideration. Additionally, there are other situations in which microsurgery may be a preferred option, including growing cystic tumors, secondary trigeminal neuralgia, or refractory dizziness [10•]; notably, the latter remains controversial. Patient preference is also an important consideration as some patients may experience irreconcilable anxiety due to the presence of an intracranial tumor. Despite the challenges of operating on a previously irradiated tumor, surgery continues to be the pre-eminent salvage option when radiosurgery fails to control tumor growth [11]. Finally, some advocate for proactive surgical resection of small favorable tumors with good preoperative hearing in order to preserve long-term serviceable hearing [12].

Between the three most commonly employed approaches, there are inherent differences that should be considered during treatment planning (Table 1). The retrosigmoid craniotomy has continued to be one of the most commonly used approaches for tumor excision, favored due a relatively high degree of posterior fossa visualization and the possibility of hearing preservation. However, it can require prolonged cerebellar retraction and offers limited visualization of the internal auditory canal without intradural, transmeatal drilling. The middle cranial fossa technique also permits hearing preservation for small, laterally based tumors with less than approximately $5 \mathrm{~mm}$ of posterior fossa extension. Some disadvantages of this approach include temporal lobe retraction and potentially a less favorable orientation of the intrameatal segment of the facial nerve for tumor dissection. The translabyrinthine approach offers adequate exposure for the removal of large 
Table 1 Advantages and disadvantages of three commonly used surgical approaches to vestibular schwannomas

\begin{tabular}{|c|c|c|c|}
\hline & Translabyrinthine (TL) & Retrosigmoid (RS) & Middle cranial fossa (MCF) \\
\hline \multirow[t]{3}{*}{ Advantages } & $\begin{array}{l}\text { Early exposure of the lateral internal } \\
\text { auditory canal }\end{array}$ & Wide exposure of the posterior fossa & Hearing preservation possibility \\
\hline & $\begin{array}{l}\text { Drilling and bleeding during approach is } \\
\text { extracranial }\end{array}$ & Hearing preservation possibility & $\begin{array}{l}\text { Early exposure of the lateral internal } \\
\text { auditory canal }\end{array}$ \\
\hline & Does not require retraction of brain & $\begin{array}{l}\text { Early identification of the facial nerve } \\
\text { root exit zone }\end{array}$ & \\
\hline \multirow[t]{3}{*}{ Disadvantages } & Ipsilateral deafness & Cerebellar retraction & Limited exposure of the brainstem \\
\hline & Neurosurgeon unfamiliarity & $\begin{array}{l}\text { Intradural drilling to access internal } \\
\text { auditory canal }\end{array}$ & Temporal lobe retraction \\
\hline & & Headache & \\
\hline
\end{tabular}

tumors and tumors extending into the fundus of the internal auditory canal [13]. It also permits identification of the facial nerve distal to the tumor. However, this approach sacrifices residual ipsilateral hearing, may be less familiar to neurosurgeons, and generally requires a separate surgical site in order to harvest an adipose graft for closure.

When microsurgical resection is sought, it is important to consider that that there are certain unavoidable risks. As with any surgical procedure, surgical site infection can occur in the post-operative period, and every effort must be taken to ensure the integrity of the sterile field. Stroke, seizure, cranial nerve injury, cerebrospinal fluid fistula, meningitis, and death are risks of intracranial surgery. In addition, hearing loss, vestibulopathy, facial nerve weakness, and lower cranial neuropathy are especially relevant when operating in the cerebellopontine angle.

Measures can be taken by the surgical team to mitigate some of these risks. With particular regard to seventh and eighth cranial nerve injury, intraoperative monitoring has proven to be a useful adjunct to microsurgical technique. Bipolar electrodes can be placed into the orbicularis oris and oculi muscles and an accompanying stimulation probe can be used to assist with facial nerve identification and prognostication. Some have suggested that the stimulus threshold and resulting discharge amplitude at the conclusion of the resection can be used to predict postoperative facial nerve function $[14,15,16 \cdot]$, though the accuracy of these measurements is not assured [17•]. When hearing preservation is attempted, intraoperative monitoring with either brainstem auditory evoked response (BAER) or direct eighth nerve monitoring (DENM) can be used [18, 19].

\section{Surgical Highlights}

Key elements of the three aforementioned surgical approaches are listed below, and our preferred incision styles are diagramed in Fig. 1. The technique for tumor dissection does not vary among the three approaches and, in general, involves meticulous isolation of the capsule and devascularization of the tumor in progressive stagesdeveloping a plane between the tumor and adjacent structures.

\section{Retrosigmoid Approach}

The retrosigmoid approach remains a mainstay of posterior fossa surgery and represents a modification of the standard suboccipital craniotomy, which provides exposure to the lateral cerebellum and cerebellopontine angle. The patient's head is ideally turned parallel to the floor or slightly past parallel. To facilitate adequate venous outflow, the patient is placed into a modified park bench position. The head is then secured using a Mayfield clamp. A curvilinear incision is made approximately three fingerbreadths behind the postauricular sulcus, extending into the neck inferiorly. Once the periosteum has been incised, the sternocleidomastoid muscle can be partially divided and retracted to provide inferior exposure. Following circumferential subperiosteal elevation, the craniotomy begins with a burr hole created over the asterion, which is the intersection of the lambdoid, squamosal, and occipitomastoid sutures. The transverse sinus and sigmoid sinus junction can usually be found beneath this landmark. Once the confluence of these sinuses is identified, a circular craniotomy is developed using a high speed burr that traces the posterior margin of the sigmoid sinus, inferior margin of the transverse sinus, and measures approximately $4 \mathrm{~cm}$ in diameter. For larger tumors the craniotomy can be enlarged to incorporate the foramen magnum. Any exposed petrous air cells are meticulously sealed with bone wax to prevent postoperative cerebrospinal fluid leak. An initial dural opening in the vicinity of the cisterna magna is made to allow for controlled egress of cerebrospinal fluid, which provides relaxation of the underlying cerebellum. A dural opening is then fashioned by raising separate superior and lateral leaflets extending all the way to the transverse and 


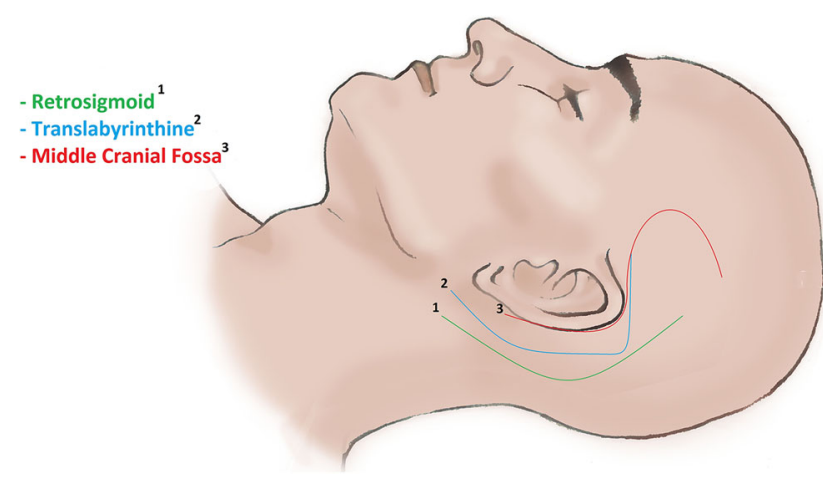

Fig. 1 Skin incisions for the retrosigmoid, translabyrinthine, and middle cranial fossa approaches

sigmoid sinuses, thereby allowing for clear visualization of the tentorium and posterior petrous temporal dura. In the case of larger tumors, a self-retaining retractor blade mounted on a Greenberg clamp can also be utilized to gently elevate the lateral cerebellum posteriorly. Further dissection of the cerebellopontine cistern superiorly will then reveal the tumor capsule and continuing arachnoid lysis toward the tentorium will then allow for optimal conditions to begin tumor dissection. Stimulation of the capsular surface and visualization for an aberrant seventh nerve is important at this stage. For smaller tumors, an initial attempt can be made to identify the root exit zone of CN VII and VIII, whereas for larger tumors, lateral debulking is performed followed by transmeatal drilling to reveal the contents of the internal auditory canal.

Once tumor resection is completed, it is critical to irrigate out blood products from the intracranial space. Meticulous hemostasis must be obtained with careful attention paid to the lateral cerebellar surface. Finally, a duraplasty is performed with our preference being to utilize a synthetic dural substitute to provide a watertight dural closure. Any exposed bony air-cells are re-waxed. The craniotomy bone flap is re-plated using a low profile, titanium plating system. The procedure ends with a multilayer, watertight closure of the surgical site.

\section{Translabyrinthine Approach}

The translabyrinthine approach utilizes standard otologic surgical techniques to exploit the close anatomic relationship of the vestibular system, internal auditory canal, and posterior fossa. The patient is placed in a supine position with the head turned away. The procedure begins with a postauricular skin incision, designed to generously expose to the tegmen superiorly, sigmoid sinus posteriorly, and mastoid tip inferiorly. Once a periosteal cut has been made, subperiosteal dissection is carried anteriorly to the level of the bony external auditory canal. The temporal bone is then drilled with the intention of eventually exposing the internal auditory canal and the posterior fossa. While drilling, we routinely decompress the sigmoid sinus and dura of the middle and posterior cranial fossae to maximize exposure. Additionally, bone is carefully thinned over the mastoid portion of the facial nerve and jugular bulb for this same purpose. As drilling proceeds medially, the endolymphatic sac and duct can be identified over the posterior fossa dura, and the superior petrosal sinus can be identified within the sinodural angle. A labyrinthectomy is performed with care to avoid the fallopian canal. Of note, if additional anterior exposure is required, a transotic or transcochlear approach can be performed after isolation of the fallopian canal in the tympanic and mastoid segments [20]. Once the internal auditory canal dura has been identified, inferior and superior troughs are drilled so that $180^{\circ}-270^{\circ}$ of exposure is obtained. When a high jugular bulb compromises exposure to the caudal end of the internal auditory canal, some surgeons will place successive layers of ovoid bone wax to compress the vein [21]. Microdissection of the tumor commences once the internal auditory canal and posterior fossa dura have been opened to sufficiently reveal the extent of the tumor.

Following tumor excision, the middle ear space and Eustachian tube are obliterated with free muscle, fat and fascia grafts, and an additional fat graft is used to further obliterate the resulting defect in the posterior petrous temporal bone. Dural substitute or an autologous fascia graft can also be used to cover the posterior fossa and internal auditory canal. Additionally, our center has adopted the use of an absorbable mesh for cranioplasty following translabyrinthine tumor resection. The mesh acts as a buttress for the fat graft, and our initial experience suggests that it can reduce the rate of postoperative pseudomeningocele formation and cerebrospinal fluid rhinorrhea. The surgical site is then closed in a multilayer, watertight fashion and the finally the tympanic membrane is examined to ensure that it was not violated during middle ear packing.

\section{Middle Cranial Fossa Approach}

The middle cranial fossa approach uses a subtemporal, extradural approach along the middle cranial fossa floor to expose the internal auditory canal from above. The patient is positioned similarly to the translabyrinthine approach. A lumbar drain may be employed to facilitate brain relaxation and minimize retraction. A pre- or postauricular incision extends in a curvilinear fashion within the hairline, centered near the ear canal. An approximately $4 \mathrm{~cm} \times 4 \mathrm{~cm}$ craniotomy is centered superior to the external auditory canal, and the bone flap is preserved for cranioplasty at the end of the case. Subtemporal dissection proceeds from 
lateral to medial and posterior to anterior in order to clearly identify the petrous ridge and the arcuate eminence and to reduce the risk of traction injury to the facial nerve from greater superficial petrosal nerve (GSPN) pull. Dissection identifies the foramen spinosum anteriorly and is careful to avoid the geniculate ganglion, which can be dehiscent. With these structures outlined, internal auditory canal can be identified by bisecting an angle drawn from the arcuate eminence and the GSPN. Drilling generally commences anteromedially near the petrous ridge to avoid injury to the labyrinth or facial nerve. Once the troughs are adequately developed and the IAC is decompressed, the dura is opened and tumor dissection is performed.

Following tumor excision, bone wax is applied to seal any of the air cells opened while drilling, and a free muscle graft is applied to fill the dural defect of the internal auditory canal. The temporal lobe is gently relaxed and the bone flap is secured over the craniotomy site with a low profile, titanium mini-plating system. The surgical site is then closed in a multilayer, watertight fashion.

\section{Surgical Outcomes}

\section{Facial Nerve Function}

Maximizing facial nerve outcomes is a goal of tumor treatment, regardless of the approach. In a 2011 report, Falcioni et al. [22] compared facial nerve outcomes across surgical approaches for 439 tumors that extended less than $1 \mathrm{~cm}$ into the cerebellopontine angle. No statistical difference was identified between the translabyrinthine and retrosigmoid approaches, but both were statistically more likely to yield better facial nerve outcomes at 1 year following resection than the middle cranial fossa approach. A recent metanalysis comparing postoperative morbidity among the three surgical approaches found that facial nerve function at the last follow-up was dependent on both tumor size and approach [23•]. With tumors less than $1.5 \mathrm{~cm}$ in the greatest diameter, the middle fossa approach resulted in the fewest cases of facial weakness worse than HouseBrackmann grade 3, whereas tumors larger than $1.5 \mathrm{~cm}$ had the lowest incidence of similar postoperative facial nerve weakness using the retrosigmoid approach. Conflicting reports such as these make conclusive declarations of superiority difficult when comparing surgical approaches in terms of facial nerve function. However, the data suggests more definitively that facial nerve outcomes depend on preoperative tumor size, with preservation of preoperative function more challenging with larger tumors. Additionally, surgeon experience plays a role in postoperative facial nerve function, regardless of approach [24, $25,26 \bullet]$.
Deliberately incomplete tumor resection, in conjunction with any surgical approach, is an emerging but controversial concept that may improve facial nerve outcomes in certain settings. Though complete tumor resection is preferred when possible, tumor capsule can be left in order to preserve cranial nerve integrity. When considering this technique, it is important to note that a larger amount of residual tumor may correlate with an increased risk of continued tumor growth following resection. Schwartz et al. [27•] recently elaborated on the potential risks and benefits of incomplete resection by reporting a 10-year experience comparing the results of gross total resection (GTR, all visible tumor removed), near-total resection (NTR, less than $1 \mathrm{~cm}$ remained clinically or radiographically), and subtotal resection (STR, greater than $1 \mathrm{~cm}$ remained clinically or radiographically). STR and NTR resulted in significantly improved postoperative facial nerve function and significantly higher rates of residual tumor growth requiring salvage surgery at an average follow-up of 3.7 years. Porter et al. [28•] describe an improved rate of facial nerve function compared to previous reports for tumors larger than $3 \mathrm{~cm}$ using an initial STR followed by a planned, second stage resection via a different approach 4-6 months later. Though the concept of STR theoretically compromises a core principle of oncologic surgery, published reports are thus far encouraging that the presence of residual, benign disease may offer a lesser degree of morbidity than facial nerve injury. However, long-term follow-up data will be needed to help validate this perspective given the possibility of recurrent tumor growth over time.

\section{Hearing Preservation}

Rates of hearing preservation associated with microsurgery have become increasingly scrutinized in recent years, particularly in light of what appears to be possible following radiation [29•]. In order to clearly define degrees of hearing preservation and loss, a Committee on Hearing and Equilibrium, though the American Academy of Otolaryngology-Head and Neck Surgery (AAO-HNS), established a four-tiered classification system based on speech discrimination and pure tone averages [30]. The GardnerRobertson classification system was also created for this purpose [31], and these two systems are described in Table 2. When comparing the three major surgical approaches to VS with regard to hearing preservation, the translabyrinthine approach intrinsically eliminates all residual hearing and is thus reserved for cases in which no serviceable hearing exists or it is not believed that hearing can be realistically preserved with microsurgical dissection [32]. The middle cranial fossa and the retrosigmoid approaches are both routinely used in attempts to preserve 
Table 2 AAO-HNS and Gardner-Robertson hearing classification scales

\begin{tabular}{ll}
\hline AAO-HNS & Gardner-Robertson \\
\hline Class A & Class I (good) \\
Discrimination 70-100 $\%$ & Discrimination 70-100 \% \\
PTA $<30 \mathrm{~dB}$ & PTA $<30 \mathrm{~dB}$ \\
Class B & Class II (serviceable) \\
Discrimination 50-69\% & Discrimination $>50-69 \%$ \\
PTA 31-50 dB & PTA 31-50 dB \\
Class C & \\
Discrimination 50-69 $\%$ & \\
PTA $>$ 50 dB & \\
Class D & \\
Discrimination $<50 \%$ & Class III (nonserviceable) \\
Any PTA & Discrimination 5-49\% \\
& PTA 51-90 dB \\
& Class IV (poor) \\
& Discrimination 1-4 \% \\
& PTA > 91 dB \\
& Class V (none) \\
& Discrimination 0 \% \\
& PTA none \\
\hline
\end{tabular}

hearing, and current data would suggest that in experienced hands, the choice of surgical approach cannot alone be used to explain differences in outcome $[33 \bullet, 34]$. This finding appears to be durable over time, as well $\left[35^{\bullet}, 36 \bullet\right.$. When counseling patients about microsurgery, preoperative hearing levels, and tumor-specific factors may be the most appropriate predictors of hearing preservation [25]. Changes in the intensity of cochlear perilymph, as demonstrated on fluid-attenuated inversion recovery (FLAIR) sequence of MRI, may also prove to be useful in predicting the likelihood of hearing loss associated with tumor management [37•].

\section{Cerebrospinal Fluid Leak}

Cerebrospinal fluid leakage following microsurgical resection of VS occurs in approximately $5-12 \%$ of cases, and the relative risk among the three different approaches has been previously evaluated. When a postoperative cerebrospinal fluid leakage occurs, it is generally through one of two mechanisms: leakage around the craniotomy site leading to the formation of a pseudomeningocele or leakage into the temporal bone and passage through the Eustachian tube resulting in rhinorrhea. Postoperative CSF leak increases the risk of meningitis as well as the duration of hospital stay and the cost of treatment [38•, 39]. For these reasons, care must be taken at the conclusion of any surgical resection to close potential communications that could permit a leak. Small air cells in the temporal bone are obliterated with bone wax, and dural defects are closed in a watertight fashion, when possible, or obliterated with autologous fat, fascia or muscle. In 2004, a metanalysis by Selesnick et al. [40] reported leak rates of $10.6 \%$ after either the retrosigmoid or the middle cranial fossa approach and $9.5 \%$ after the translabyrinthine approach with no statistically significant difference between the three. More recently, we reported the cumulative experience of the Otology Group between 1970 and 2010 and came to a similar conclusion with the additional note of an increased incidence of leakage associated with combined approaches [41].

Over the past decade, modifications in surgical technique have been described that have reportedly led to reductions in the occurrence of cerebrospinal fluid leakage. Different authors have advocated for measures such as routine mastoid obliteration during retrosigmoid approaches in order to more effectively seal the temporal bone and tympanic cavity [42] and the use of sealants to reinforce dural repair [43•]. Cranioplasty alterations have also been described. Manjilla et al. [44-] reported on the use of a titanium mesh with overlying hydroxyapatite cement, noting no instances of CSF leak in 42 consecutive resections. However, enthusiasm for the use of hydroxyapatite in this setting has been mixed given the possibility of associated complications [45]. Absorbable mesh cranioplasty has also been described, but no significant reduction in cerebrospinal fluid leakage was demonstrated [46].

\section{Tumor Recurrence}

Recurrent tumor growth represents a challenging dilemma in the contemporary management of VS. Treatment failure, either following surgery or radiation, can lead to tumors with a relatively advanced rate of growth and worse functional outcomes following salvage surgery [47•, 48•, 49•]. After microsurgical resection for sporadic tumors, recurrence almost certainly results from the presence of residual tumor, frequently in areas where visualization can be difficult such as the fundus of the internal auditory canal. Areas where tumor is adherent to the facial nerve, brainstem, or vasculature are also suspect. In terms of the different surgical approaches to tumors, it does not appear that one approach is more likely to lead to recurrence, though the importance of adequate exposure to the lateral extent of tumor growth in the internal auditory canal is generally emphasized [50•]. When tumor remains following resection, subsequent growth appears to correlate with the size of the remainder. Carlson et al. [51••] demonstrated a significant increase in the rate of residual tumor growth following STR compared with NTR and GTR, particularly when nodular enhancement was quantified on MRI as 
greater than $15 \mathrm{~mm}$ in diameter or $0.4 \mathrm{~cm}^{3}$ in volume. For this reason, we believe that serial post-operative MRI is imperative to monitor for new tumor growth in patients who have undergone incomplete surgical resection. In this setting, our center advocates for routine post-operative MRI within $24 \mathrm{~h}$ of resection to serve as a baseline, 1 year following resection and every 1-2 years thereafter until stability has been established. At that point, radiographic follow-up can usually be extended to 3-5 year intervals. When GTR has been achieved, the follow-up MRI intervals are generally longer, though the exact schedule can vary between institutions $[52,53 \bullet]$.

\section{Headache}

Post-operative headaches can significantly influence patient quality of life following tumor resection. Though the mechanisms by which patients experience headaches are likely variable, attempts have been made to correlate the persistence of this symptom with the style of surgical approach. Traditionally, the retrosigmoid approach has been associated with a higher incidence of post-operative headaches [23]. Many reports have identified different aspects of the retrosigmoid approach as contributors to post-operative headaches, such as the division of nuchal musculature and nerves, the adhesion of nuchal musculature to dura, dural irritation following suture closure, and the creation of intradural bone dust after transmeatal drilling [54]. Addressing some of these potential etiologies at the time of surgery may help to reduce the incidence of postoperative headache. If possible, we would advocate for the avoidance of tight dural closure, for the use of meticulous irrigation to clean bone dust from the intradural space, and for the avoidance of chemical irritants that can cause intracranial inflammation.

\section{Future Consideration: The Endoscope-Assisted Approach}

The application of the endoscope to posterior fossa surgery may ultimately prove to be a valuable adjunct to microsurgical resection. Reports of endoscopic-assisted techniques for VS surgery are emerging [55•], but superiority over existing microscopic techniques is not widely accepted. While the microscope provides the visualization necessary for complete tumor resection in most cases, it is possible that future work could demonstrate the utility of a high-definition view from an endoscope when attempting to see structures out of a direct line-of-sight or when attempting to exploit an otherwise obscure plane of dissection between two structures.

\section{Conclusion}

Microsurgical resection continues to be an important part of the treatment algorithm for VS. There are intrinsic differences in the three main surgical approaches used for tumor resection, and in general, the most appropriate approach depends on the tumor, the patient, and the surgical team. As the emphasis on treatment morbidity and patient quality of life continues to evolve, we predict that the importance of multidisciplinary skull base centers in contemporary VS management will become increasingly apparent.

Acknowledgments The authors would like to acknowledge and thank Jennifer Best, M.D. for her assistance with the illustration in Fig. 1.

\section{Compliance with Ethics Guidelines}

Conflict of Interest David S. Haynes is a consultant for Advanced Bionics, MED-EL GmbH, Cochlear, Grace Medical, Stryker and Synthes. Reid Thompson, Alex Sweeney, Matthew Carlson and Moneeb Ehtesham declare that they have no conflict of interest.

Human and Animal Rights and Informed Consent No human or animal studies performed by the authors. This article does not contain any studies with human or animal subjects performed by any of the authors.

\section{References}

Papers of particular interest, published recently, have been highlighted as:

- Of importance

•- Of major importance

1. Cushing H. Tumors of the nervus acusticus and the syndrome of the cerebellopontine angle. Philadelphia and London: W.B. Saunders Company; 1971.

2. Machinis TG, Fountas KN, Dimopoulos V, Robinsion JS. History of acoustic neurinoma surgery. Neurosurg Focus. 2005;18(4):1-4.

3. Stangerup SE, Tos M, Caye-Thomasen P, Tos T, Klokker M, Thomasen J. Increasing annual incidence of vestibular schwannoma and age at diagnosis. J Laryngol Otol. 2004;118:622-7.

4. Stangerup SE, Caye-Thomasen P, Tos M, Thonsen J. The natural history of vestibular schwannoma. Otol Neurotol. 2006;24(7): $547-52$.

5. - Huang X, Caye-Thomassen P, Stangerup SE. Spontaneous tumour shrinkage in 1261 observed patients with sporadic vestibular schwannoma. J Laryngol Otol. 2013;127(8):739-43. This study evaluates the incidence of spontaneous, radiographic tumor shrinkage in patients who are being observed with a "wait-andscan" strategy.

6. • Lau T, Olivera R, Miller T Jr, Downes K, Danner C, van Lovern HR, Agazzi S. Paradoxical trends in the management of vestibular schwannoma in the United States. J Neurosurg. 2012;117:514-9. This study is an overview of the SEER database as it relates to vestibular schwannoma management strategies. 
7. • Link MJ, Driscoll CLW, Foote RL, Pollock BE. Radiation therapy and radiosurgery for vestibular schwannomas. Otolaryngol Clin N Am. 2012;45(2):353-66. This paper is a comprehensive overview of radiation-based therapies for vestibular schwannomas.

8. • Robinett ZN, Walz PC, Miles-Markley B, Moberly AC, Welling DB. Comparison of long term quality-of-life outcomes in vestibular schwannoma patients. Otolaryngol Head Neck Surg. 2014;150(6):1024-32. This study evaluates long-term quality of life amongst the three major treatment strategies for vestibular schwannomas.

9. • Carlson ML et al. Long-term quality of life in patients with vestibular schwannoma: an international multicenter cross-sectional study comparing microsurgery, stereotactic radiosurgery, observation, and non-tumor controls. J Neurosurg (accepted). This study also evaluates long-term quality of life amongst the three major treatment strategies for vestibular schwannomas.

10. - Williams BJ, Xu Z, Salvetti DJ, McNeill IT, Lamer J, Sheehan JP. Gamma Knife surgery for large vestibular schwannomas: a single-center retrospective case-matched comparison assessing the effect of lesion size. J Neurosurg. 2013;119(2):463-71. This study evaluates tumor size and treatment responses following gamma knife radiation.

11. Slattery WH. Microsurgery after radiosurgery or radiotherapy for vestibular schwannomas. Otolaryngol Clin North Am. 2009;42:707-15.

12. Meyer TA, Canty PA, Wilkinson EP, Hansen MR, Rubinstein JT, Gantz BJ. Small acoustic neuromas: surgical outcomes versus observation or radiation. Otol Neurotol. 2006;27(3): 380-92.

13. Mamikoglu B, Wiet RJ, Esquivel CR. Translabyrinthine approach for the management of large and giant vestibular schwannomas. Otol Neurotol. 2002;23(2):224-7.

14. Isaacson B, Kileny PR, El-Kashlan HK. Prediction of long-term facial nerve outcomes with intraoperative nere monitoring. Otol Neurotol. 2005;26(2):270-3.

15. Neff BA, Ting K, Dickinson SL, Welling DB. Facial nerve monitoring parameters as a predictor of postoperative facial nerve outcomes after vestibular schwannoma resection. Otol Neurotol. 2005;26(4):728-32.

16. - Arnolder C, Mick P, Pirouzmand F, Houlden D, Lin VY, Nedzelski JM, Chen JM. Facial nerve prognostication in vestibular schwannoma surgery: the concept of percent maximum and its predictability. Laryngoscope. 2013;123(10):2533-8. This study evaluates the prognostic value of neurophysiologic testing for the facial nerve in vestibular schwannoma resection.

17. - Carlson ML, Van Abel KM, Schmitt WR, Driscoll CL, Neff BA, Link MJ. The anatomically intact but electronically unresponsive facial nerve in vestibular schwannoma surgery. Neurosurgery. 2012;71(6):1125-30. This study also evaluates the prognostic value of neurophysiologic testing for the facial nerve in vestibular schwannoma resection.

18. Phillips DJ, Kobylarz EJ, De Peralta ET, Stieg PE, Selesnick SH. Predictive factors of hearing preservation after surgical resection of small vestibular schwannomas. Otol Neurotol. 2010;31(9):1463-8.

19. Danner C, Mastrodimos B, Cueva RA. A comparison of direct eighth nerve monitoring and auditory brainstem response in hearing preservation surgery for vestibular schwannoma. Otol Neurotol. 2004;25(5):826-32.

20. Chen JM, Fisch U. The transotic approach in acoustic neuroma surgery. J Otolaryngol. 1993;22(5):331-6.

21. Moffat DA, Quaranta N, Chang P. Management of the high jugular bulb in translabyrinthine surgery. Laryngoscope. 2003;113(3):580-2.

22. Falcioni M, Fois P, Talbah A, Sanna M. Facial nerve function after vestibular schwannoma surgery. J Neurosurg. 2011;115(4):820-6.
23. •• Ansari SF, Terry C, Cohen-Gadol AA. Surgery for vestibular schwannomas: a systematic review of complications by approach. Neurosurg Focus. 2012;33(3):E14. This study is a metanalysis of different outcomes following microsurgery.

24. Welling DB, Slater PW, Thomas RD, McGregor JM, Goodman JE. The learning curve in vestibular schwannoma surgery. Am J Otol. 1999;20(5):644-8.

25. Isaacson B, Telian SA, El-Kashlan HK. Facial nerve outcomes in middle cranial fossa vs translabyrinthine approaches. Otolaryngol Head Neck Surg. 2005;133(6):906-10.

26. - Moffat DA, Parker RA, Hardy DG, Macfarlane R. Factors affecting final facial nerve outcome following vestibular schwannoma surgery. J Laryngol Otol. 2014;128(5):406-15. This study assesses different factors that may contribute to facial nerve outcomes following vestibular schwannoma resection.

27. • Schwartz MS, Kari E, Strickland M, Berliner K, Brackmann DE, House JW, Friedman RA. Evaluation of the increased use of partial resection of large vestibular schwannomas: facial nerve outcomes and recurrence/regrowth rates. Otol Neurotol. 2013;34(8):1456-64. This study evaluates the effectiveness of subtotal tumor resection as it relates to facial nerve outcome and tumor regrowth.

28. - Porter RG, LaRouere MJ, Kartush JM, Bojrab DI, Pieper DR. Improved facial nerve outcomes using an evolving treatment method for large acoustic neuromas. Otol Neurotol. 2013;34(3):304-10. This study evaluates staged resection as a treatment approach for vestibular schwannomas.

29. - Maniakas A, Saliba I. Microsurgery versus stereotactic radiation for small vestibular schwannomas: a meta-analysis of patients with more than 5 years' follow-up. Otol Neurotol. 2012;33(9):1611-20. This study is a meta-analysis that compares radiation versus microsurgery outcomes for vestibular schwannoma patients.

30. Committee on Hearing and Equilibrium guidelines for the evaluation of hearing preservation in acoustic neuroma (vestibular schwannoma): American Academy of Otolaryngology-Head and Neck Surgery Foundation, Inc. Otolaryngol Head Neck Surg. 1995;113:179-80.

31. Gardner G, Robertson JH. Hearing Preservation in unilateral acoustic neuroma surgery. Ann Otol Rhinol Laryngol. 1988;97(1):55-66.

32. Yates PD, Jackler RK, Satar B, Pitts LH, Oghalai JS. Is it worthwhile to attempt hearing preservation in larger acoustic neuromas. Otol Neurotol. 2003;24(3):460-4.

33. - Rabelo de Freitas M, Russo A, Sequino G, Piccirillo E, Sanna M. Analysis of hearing preservation and facial nerve function for patient undergoing vestibular schwannoma surgery: the middle cranial fossa approach versus the retrosigmoid approach-personal experience and literature review. Audiol Neurootol. 2012;17(2):71-81. This study compares the middle fossa and retrosigmoid approaches to vestibular schwannoma resection on the grounds of hearing preservation and facial nerve function.

34. Sameshima T, Fukushima T, McElveen JT Jr, Friedman AH. Critical assessment of operative approaches for hearing preservation in small acoustic neuroma surgery: retrosigmoid vs middle fossa approach. Neurosurgery. 2010;67(3):640-4.

35. - Wang AC, Chinn SB, Than KD, Arts HA, Telian SA, ElKashlan HK, Thompson BG. Durability of hearing preservation after microsurgical treatment of vestibular schwannoma using the middle cranial fossa approach. J Neurosurg. 2013;119(1):131-8. This study evaluates long-term hearing outcomes following middle fossa tumor resection.

36. - Yamakami I, Ito S, Higuchi Y. Retrosigmoid removal of small acoustic neuroma: curative tumor removal with preservation of function. J Neurosurg. 2014;4:1-10. This study discusses the experience of a center with hearing preservation surgery via the retrosigmoid approach. 
37. - Miller ME, Mafee MF, Bykowski J, Alexander TH, Burchette RJ, Mastrodimos B, Cueva RA. Hearing preservation and vestibular schwannoma: intracochlear FLAIR signal relates to hearing level. Otol Neurotol. 2014;35:348-52. This study evaluates the use of MRI as a prognostic indicator when undertaking hearing preservation resection.

38. - Allen KP, Isaacson B, Kutz JW, Purcell PL, Roland PS. The association of meningitis with postoperative cerebrospinal fluid fistula. J Neurol Surg B. 2012;73(6):401-4. This study evaluates rates of meningitis in patients with CSF leaks following surgery.

39. Grotenhuis JA. Costs of postoperative cerebrospinal fluid leakage: 1-year, retrospective analysis of 412 consecutive nontrauma cases. Surg Neurol. 2005;64(6):490-3.

40. Selesnick SH, Liu JC, Jen A, Newman J. The incidence of cerebrospinal fluid leak after vestibular schwannoma surgery. Otol Neurotol. 2004;25(3):387-93.

41. Mangus BD, Rivas A, Yoo MJ, Alvarez J, Wanna GB, Haynes DS, Bennett ML. Management of cerebrospinal fluid leaks after vestibular schwannoma surgery. Otol Neurotol. 2011;32(9):1525-9.

42. Falcioni M, Romano G, Aggarwal N, Sanna M. Cerebrospinal fluid leak after retrosigmoid excision of vestibular schwannomas. Otol Neurotol. 2008;29(3):384-6.

43. - Kawai H, Nakagawa I, Nishimura F, Motoyama Y, Park YS, Nakamura M, Nakase H, Suzuki S, Ikada Y. Effectiveness of a new gelatin sealant system for dural closure. Neurol Res. 2014. This study evaluates a novel method of closure following intracranial surgery.

44. - Manjilla S, Weidenbecher M, Semaan MT, Megerian CA, Bambakidis NC. Prevention of postoperative cerebrospinal fluid leaks with multilayered reconstruction using titanium meshhydroxyapatite cement cranioplasty after translabyrinthine resection of acoustic neuroma. J Neurosurg. 2013;119(1):113-20. This study also evaluates a novel method of closure following intracranial surgery.

45. Benson AG, Djalilian HR. Complications of hydroxyapatite bone cement reconstruction of retrosigmoid craniotomy: two cases. Ear Nose Throat J. 2009;88(11):E1-4.

46. Hillman TA, Shelton C. Resorbable plate cranioplasty after the translabyrinthine approach. 2012. Otol Neurotol. 2011;32(7):1171-4.

47. - Chen Z, Prasad SC, Di Lella F, Medina M, Piccirillo E, Taibah A, Russo A, Yin S, Sanna M. The behavior of residual tumors and facial nerve outcomes after incomplete excision of vestibular schwannomas. J Neurosurg. 2014;120(6):1278-87. This study analyzes the postoperative outcomes of patients who had subtotal tumor resections.

48. - Gerganov VM, Giordano M, Samii A, Samii M. Surgical treatment of patients with vestibular schwannomas after failed previous radiosurgery. J Neurosurg. 2012;116(4):713-20. This study evaluates the effect of radiosurgery on subsequent attempts at surgical resection.

49. - Husseini ST, Piccirillo E, Taibah A, Almutair T, Swquino G, Sanna M. Salvage surgery of vestibular schwannoma after failed radiotherapy: the Gruppo Otologico experience and review of the literature. Am J Otolaryngol. 2013;34(2):107-14. This study also evaluates the effect of radiosurgery on subsequent attempts at surgical resection.

50. - Ahmad RA, Sivalingam S, Topsakal V, Russo A, Taibah A, Sanna M. Rate of recurrent vestibular schwannoma after total removal via different surgical approaches. Ann Otol Rhinol Laryngol. 2012;121(3):158-61. This study analyzes each surgical approach with regard to the incidence of subsequent tumor recurrence.

51. • Carlson ML, Van Abel KN, Driscoll CL, Neff BA, Beatty CW, Lane JI, Castner ML, Lohse CM, Link MJ. Magnetic resonance imaging surveillance following vestibular schwannoma resection. Laryngoscope. 2012;122(2):378-88. This study evaluates the risk of residual tumor growth based on postoperative MRI findings.

52. Bennett ML, Jackson CG, Kaufmann R, et al. Postoperative imaging of vestibular schwannomas. Otolaryngol Head Neck Surg. 2008;138(5):667-71.

53. - Tysome JR, Moffat DA. Magnetic resonance imaging after translabyrinthine complete excision of vestibular schwannomas. J Neurol Surg B. 2012;73(2):121-4. This study evaluates a postoperative imaging algorithm for tumor surveillence following microsurgical resection.

54. Schaller B, Baumann A. Headache after removal of vestibular schwannoma via the retrosigmoid approach: a long term followup study. Otolaryngol Head Neck Surg. 2003;128(3):387-95.

55. - Kumon Y, Kohno S, Ohue S, Hideaki W, Inoue A, Iwata S, Ohnishi T. Usefulness of endoscope-assisted microsurgery for removal of vestibular schwannomas. J Neurol Surg B. 2012;73(1):42-7. This study evaluates the use of endoscopic techniques during microsurgical resection of vestibular schwannomas. 